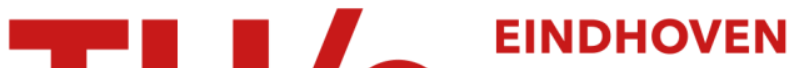 \\ UNIVERSITY OF \\ TECHNOLOGY
}

\section{Adaptive isogeometric analysis applied to an electromagnetic actuator}

Citation for published version (APA):

Friedrich, L., Gysen, B., Roes, M., \& Lomonova, E. (2019). Adaptive isogeometric analysis applied to an electromagnetic actuator. IEEE Transactions on Magnetics, 55(6), [7401104].

https://doi.org/10.1109/TMAG.2019.2900529

DOI:

10.1109/TMAG.2019.2900529

Document status and date:

Published: 18/05/2019

\section{Document Version:}

Typeset version in publisher's lay-out, without final page, issue and volume numbers

\section{Please check the document version of this publication:}

- A submitted manuscript is the version of the article upon submission and before peer-review. There can be important differences between the submitted version and the official published version of record. People interested in the research are advised to contact the author for the final version of the publication, or visit the $\mathrm{DOI}$ to the publisher's website.

- The final author version and the galley proof are versions of the publication after peer review.

- The final published version features the final layout of the paper including the volume, issue and page numbers.

Link to publication

\section{General rights}

Copyright and moral rights for the publications made accessible in the public portal are retained by the authors and/or other copyright owners and it is a condition of accessing publications that users recognise and abide by the legal requirements associated with these rights.

- Users may download and print one copy of any publication from the public portal for the purpose of private study or research.

- You may not further distribute the material or use it for any profit-making activity or commercial gain

- You may freely distribute the URL identifying the publication in the public portal.

If the publication is distributed under the terms of Article 25fa of the Dutch Copyright Act, indicated by the "Taverne" license above, please follow below link for the End User Agreement:

www.tue.nl/taverne

Take down policy

If you believe that this document breaches copyright please contact us at:

openaccess@tue.nl

providing details and we will investigate your claim. 


\title{
Adaptive Isogeometric Analysis Applied to an Electromagnetic Actuator
}

\author{
L. A. J. Friedrich ${ }^{\circledR}$, B. L. J. Gysen ${ }^{\circledR}$, M. G. L. Roes, and E. A. Lomonova \\ Department of Electrical Engineering, Electromechanics and Power Electronics, Eindhoven University of Technology, \\ 5600 MB Eindhoven, The Netherlands
}

In this paper, a magnetostatic solver for linear and nonlinear soft-magnetic material characteristics, a time-harmonic eddy current solver, and the corresponding function-based error estimates are implemented in an established adaptive isogeometric analysis framework. The simplified truncated hierarchical B-spline basis functions are investigated on the multipatch geometry of a magnetic circuit, as they offer several beneficial properties, including a drastic reduction of the number of degrees of freedom compared to the references under uniform refinement. Global error estimate and global parameters convergence are illustrated for different refinement strategies and polynomial orders. Finally, the computational effort is analyzed.

Index Terms-Adaptive methods, eddy current problem, hierarchical splines, isogeometric analysis, nonlinear characteristics.

\section{INTRODUCTION}

$\mathbf{E}$ LECTROMAGNETIC design relies on the accurate estimation of the local field quantities (electric, magnetic, stress, and temperature), the description of complex geometrical shapes, as well as the calculation of global parameters, such as force and eddy current losses. The latter might be impaired by local inaccuracies, which depend on the geometry and the employed solver. To accurately model and efficiently optimize the electromagnetic devices, it is essential to refine the discretization locally while maintaining an overall low computational effort. The concept of adaptive hierarchical meshes is particularly attractive for the simulation of multiscale problems and in the context of design optimization [1].

In this paper, an adaptive isogeometric method [2] using the simplified truncated hierarchical B-splines (THB) structure [3], [4] is applied to the modeling and analysis of an electromagnetic actuator. Both linear and nonlinear magnetostatic problems as well as a time-harmonic eddy current problem are implemented in the numerical framework [5]. Several marking strategies and polynomial orders for the THB-splines are compared. Using such an adaptive method alleviates the need for truncating the geometrical model and manually fine-tuning mesh and space parameters while ensuring faster convergence together with low sensitivity on the selected mesh refinement level and polynomial order.

\section{Problem Formulation}

The Galerkin approximations of the 2-D magnetostatic problem and the harmonic eddy current problem are solved on the C-core benchmark actuator exemplified in Fig. 1(a). The vector potential solution reduces to its scalar $z$-component, which naturally satisfies the Coulomb gauge. Essential boundary conditions are imposed weakly on all sides through a lifting and $L^{2}$ projection [5].

Manuscript received October 25, 2018; revised January 18, 2019; accepted February 15, 2019. Corresponding author: L. A. J. Friedrich (e-mail: 1.friedrich@tue.nl).

Digital Object Identifier 10.1109/TMAG.2019.2900529
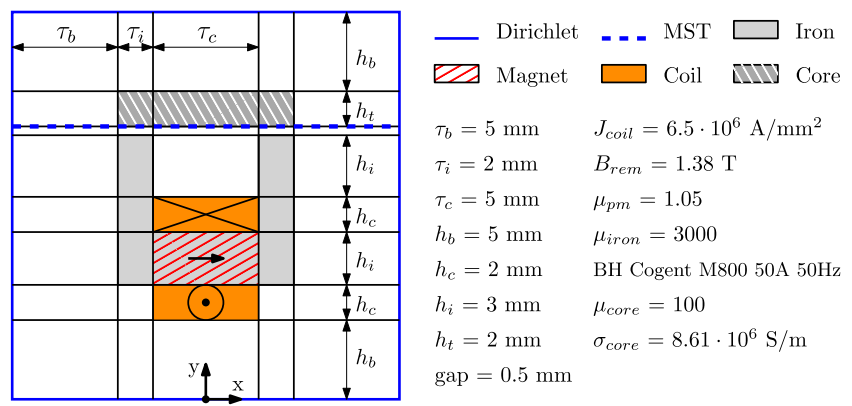

(a)

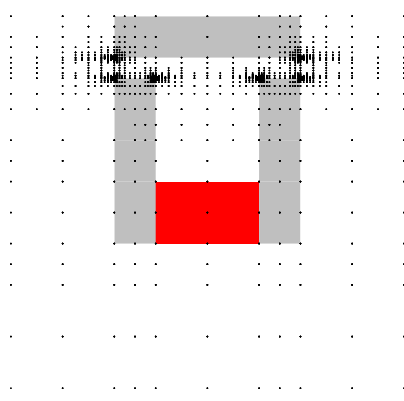

(b)

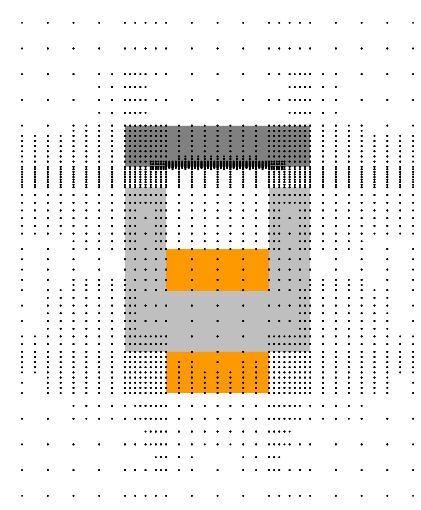

(c)
Fig. 1. (a) Benchmark topology of the C-core actuator, and optimum mesh resulting from the MS strategy with biquartic THB-splines refined up to level 6, for (b) magnetostatic and (c) magnetoharmonic problems.

For the magnetostatic problems, a permanent magnet uniformly magnetized is considered as the source of magnetic field. Furthermore, nonlinear $B-H$ characteristics are included using the Newton-Raphson method. Isotropic and nonhysteretic materials are considered in this paper although more complex material models can be included [6].

For the magnetoharmonic problem, the magnet is replaced by a coil carrying alternating current of frequency $f=1 \mathrm{MHz}$. This is done deliberately to impose a skin depth two orders or magnitude smaller than the geometrical 
dimension of the conductive core. Such challenging multiscale problem has applications in high-frequency transformers [7]. At the frequency and amplitude of the current source considered in this paper, the soft-magnetic materials are far from their saturation levels; therefore, nonlinearity has been neglected. Direct dependence of the $B-H$ curve with the frequency has not been considered. Nevertheless, nonlinear material characteristics can be included by following the harmonic balance method [8].

\section{THB-SPLINE BASIS CONSTRUCTION}

This paper relies on the construction of a nested sequence of the tensor product spline space $\left\{\mathcal{S}_{l}\right\}$, spanned by the bi-variate $\mathrm{B}$-spline basis $\left\{\mathcal{B}_{l}\right\}$ of degree $p$ and level $l$. The scalar-valued B-spline basis is formed by $\mathcal{B}_{i, l}=\left\{\beta_{i, l}, i=1, \ldots, N_{l}\right\}$, where $N_{l}$ is the dimension of the spline space. $\mathcal{Q}_{l}$ is the cell of level $l$, which corresponds to the associated Cartesian mesh. A fundamental point is the use of the child-parent structure

$$
\beta_{i, l}=\sum_{k=1}^{N_{l+1}} c_{k, l+1}\left(\beta_{i, l}\right) \beta_{k, l+1}, \quad \text { with } c_{k, l+1}\left(\beta_{i, l}\right) \geq 0 .
$$

This two-scale relationship can be written in a change of basis matrix that gather the coefficients at different levels

$$
\left(C_{l}^{l+1}\right)_{k i}=c_{k, l+1}\left(\beta_{i, l}\right) .
$$

The set $\boldsymbol{\Omega}_{\boldsymbol{n}}=\left\{\boldsymbol{\Omega}_{l}\right\}_{l=0, \ldots, n}$ is introduced as a hierarchy of strictly contained subdomains of depth $n$. A simplified version of the hierarchical basis $\tilde{\mathcal{H}}$ associated with $\boldsymbol{\Omega}_{\boldsymbol{n}}$ is constructed

$$
\begin{aligned}
\tilde{\mathcal{H}}_{0}= & \left\{\beta \in \mathcal{B}_{0}, \operatorname{supp} \beta \neq \emptyset\right\}, \\
\tilde{\mathcal{H}}_{l+1}= & \left\{\beta \in \tilde{\mathcal{H}}_{l}, \operatorname{supp} \beta \not \subset \Omega_{l+1}\right\} \cup \\
& \bigcup_{\beta \in \tilde{\mathcal{H}}_{l}, \operatorname{supp} \beta \subset \Omega_{l+1}} \mathcal{C}(\beta), \quad l=0, \ldots, n-2
\end{aligned}
$$

which only adds the children $\mathcal{C}(\beta)$ of the deactivated functions and is computationally easier to select and build than its traditional counterpart [9]. To define the truncated basis of $\beta_{i, l} \in \mathcal{B}_{l}$, the linear operator is introduced

$$
\operatorname{trunc}_{l+1}\left(\beta_{i, l}\right)=\sum_{k=1}^{N_{l+1}} c_{k, l+1}^{\tau}\left(\beta_{i, l}\right) \beta_{k, l+1}
$$

which coefficients are set to zero if $\beta_{k, l+1} \in \tilde{\mathcal{H}}_{l+1} \cap \mathcal{B}_{l+1}$. The construction of the simplified THB-spline basis $\tilde{\mathcal{T}}$ follows:

$$
\begin{aligned}
\tilde{\mathcal{T}}_{0}= & \left\{\beta \in \mathcal{B}_{0}, \operatorname{supp} \beta \neq \emptyset\right\}, \\
\tilde{\mathcal{T}}_{l+1}= & \left\{\operatorname{trunc}_{l+1}(\beta), \beta \in \tilde{\mathcal{T}}_{l} \wedge \operatorname{supp} \beta \not \subset \Omega_{l+1}\right\} \cup \\
& \left\{\beta \in \mathcal{B}_{l+1}, \operatorname{supp} \beta \subset \Omega_{l+1}\right\}, \quad l=0, \ldots, n-2 .
\end{aligned}
$$

The matrix collecting the THB coefficients $\left(C_{l}^{l+1, \tau}\right)$ is applied successively between the consecutive levels, and finally, the matrix $C_{l}$, which changes the basis from the active THB basis to $\mathcal{B}_{l}$, is assembled recursively

$$
\begin{aligned}
C_{0}^{\tau} & =J_{0}, \\
C_{l+1}^{\tau} & =\left[C_{l}^{l+1, \tau} C_{l}^{\tau}, J_{l+1}\right], \quad l=0, \ldots, n-2 .
\end{aligned}
$$

This matrix includes all the active level function coefficients designated by $J_{l+1}$. The constructed THB-spline basis restores the convex partition of unity, demonstrates reduced support and multilevel interactions, and preserves the coefficients.

The 2-D quasi-static electromagnetic problems, which are considered in this paper, can be written as a general elliptic variational problem that reads: find $u_{\tilde{\mathcal{T}}} \in H_{0}^{1}$

$$
a\left(u_{\tilde{\mathcal{T}}}, v_{\tilde{\mathcal{T}}}\right)=\langle f, v\rangle, \quad \forall v_{\tilde{\mathcal{T}}} \in H_{0}^{1}
$$

where $a(\cdot, \cdot)$ is a bilinear form and $f$ is a linear operator. Any matrix $M^{*}$, which might compose the bilinear form associated with the elliptic problem, such as stiffness, Jacobian or mass matrix, and the right-hand side $f$, are assembled per level, using $C_{l}^{\tau}$ to change of basis while preserving the coefficients

$$
M^{*}=\sum_{l=0}^{n-1}\left[\begin{array}{ll}
C_{l}^{\tau} & \mathbf{0}
\end{array}\right]^{T} M_{l}^{*}\left[\begin{array}{ll}
C_{l}^{\tau} & \mathbf{0}
\end{array}\right] .
$$

The rest of the assembly is done per patch to include the different material properties and source terms and later follows the isogeometric method structure of [5]. Each patch that composes the physical domain $\Omega_{i} \in \mathbb{R}^{2}$ is mapped to a reference computational element $\tilde{\Omega}=[0,1]^{2}$ through the parametrizations $\mathbf{F}_{i}$ and is glued through $C^{0}$ continuity at coinciding interfaces.

\section{Adaptive Isogeometric Method}

Four main modules constitute the adaptive algorithm: solve, estimate, mark, and refine. Linear and nonlinear magnetostatic and magnetoharmonic solvers have been implemented in the available numerical framework [5]. Estimators for the error can act on either the elements or the set of basis functions. Reliable and efficient a posteriori error indicators have been developed in [3] for the functions and in [4] for the elements. Function-based error estimates have been selected in this paper, together with a simplified version of the THB-splines, as they reduce the interaction between different refinement levels and, therefore, lead to sparser matrixes and a further reduction of the number of degrees of freedom (ndof). The error estimators are based on the $L^{2}$ norm of the specific problem residuals weighted by the mesh size $h_{\beta}$

$$
\epsilon_{\beta}=h_{\beta}\left(\int_{\mathbf{F}(\operatorname{supp} \beta)}\left|f-a\left(u_{\tilde{\mathcal{T}}}, u_{\tilde{\mathcal{T}}}\right)\right|^{2}\left(\beta \circ \mathbf{F}^{-1}\right)\right)^{\frac{1}{2}} .
$$

Such estimators are extended in this paper to the multipatch configurations to include the different material properties and are particularized to each solver bilinear form. In the employed framework, several simple marking strategies are available, the Guaranteed Error Reduction Strategy (GERS), the Maximum Strategy (MS), and the Global Refinement (GR), that can act on both elements and basis functions. The GERS is, in fact, equivalent to the marking strategy introduced by Dörfler [10], where the parameter $\theta=(1 / 2)$ is selected, which ensures that the sum of the error contributions of the marked entities represents more than half of the total estimated error

$$
\mathcal{M}=\left\{\beta, \sum_{\beta \in \tilde{\mathcal{T}}} \epsilon_{\beta} \geq \theta \sum_{\beta^{\prime} \in \tilde{\mathcal{T}}} \epsilon_{\beta^{\prime}}\right\} .
$$




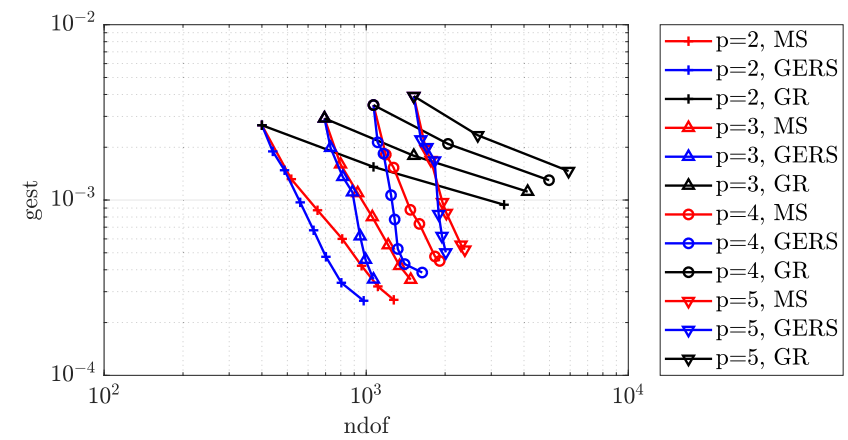

Fig. 2. Global error estimate (gest) convergence with the ndof for different adaptive refinements strategies and polynomial orders for the magnetostatic problem.

Furthermore, the MS uses the same $\theta=(1 / 2)$ parameter, which, in this case, marks the entities that exceed half of the maximum error

$$
\mathcal{M}=\left\{\beta, \epsilon_{\beta} \geq \theta \max _{\beta^{\prime} \in \tilde{\mathcal{T}}} \epsilon_{\beta^{\prime}}\right\} .
$$

The GR refines the whole mesh at each iteration, without using the error estimate. Finally, the refine module uses the advantageous properties of the THB-splines meshes and approximation spaces and has not been modified. At each level, the active cells that are contained in the support of the marked function are dyadically refined, creating an enlargement of the subdomains $\boldsymbol{\Omega}_{\boldsymbol{n}+\boldsymbol{1}}^{*}$. The marked basis functions are deactivated and replaced by their children. Details on the THB-splines refinements algorithms can be found in [2]. Analysis and proofs of the optimal local approximation properties and convergence of the error estimates are detailed in [9] using a multiscale quasi-interpolant operator.

\section{RESULTS}

In this section, the convergence of the parameters (force and eddy current losses) and global error estimate (gest) are analyzed for different marking strategies and polynomial orders, for both static and time-harmonic solvers. All the results presented in this paper have been obtained using the THBsplines of degree $p \in[2,5]$. The hierarchical meshes obtained through the adaptive refinements are shown in Fig. 1(b)-(c), for both problems. In Fig. 1(b)-(c), only the mesh corners, i.e., the intersections of the quadrilateral mesh cells, have been represented by dots, which density distribution gives the localized mesh refinement indication. The convergence of the energy-based global error estimate (gest) versus the ndof is given in Figs. 2 and 3, for the magnetostatic and the magnetoharmonic problems, respectively. Each point corresponds to an iteration of the adaptive method. The global error estimate converges for every refinement strategy with increasing slope for increasing polynomial order. The GR is the least effective strategy. It can be seen in Fig. 2 that for the eddy current problem, increasing the polynomial order $(p \geq 5)$ with a coarse mesh deteriorates the global estimate, which is related to a conditioning issue [11].

From the nonlinear magnetostatic field distribution, the attraction force is calculated through the Maxwell Stress

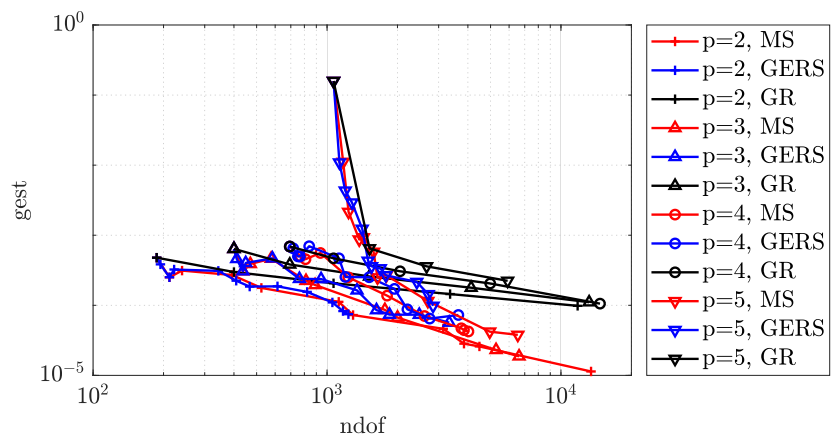

Fig. 3. Global error estimate (gest) convergence with the ndof for different adaptive refinements strategies and polynomial orders for the magnetoharmonic problem.

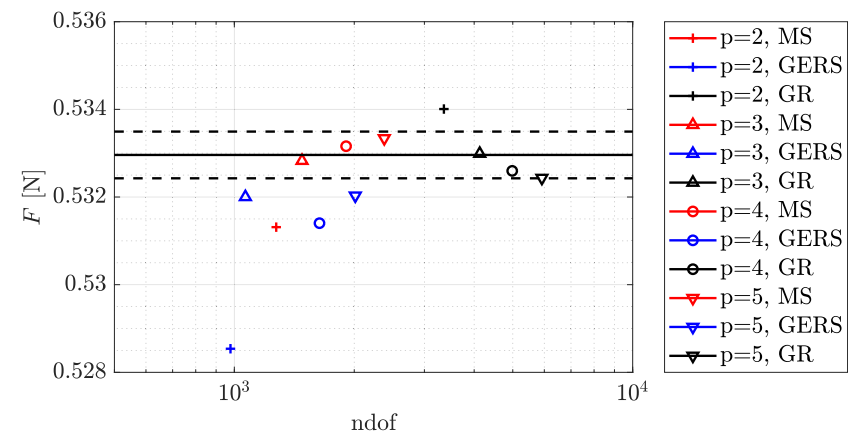

Fig. 4. Force convergence for the different refinement strategies and polynomial orders. The black line represents the value obtained from FEA with $1.42 \cdot 10^{5}$ dof, and the dashed lines represent a variation of $0.1 \%$.

Tensor on a contour surrounding the core. This contour is located on top of the air gap and on the three Dirichlet contributions, where the fields vanish exactly, therefore minimizing the numerical noise compared to an integration directly over the core-air interface. The convergence of the force is shown in Fig. 4 for different marking strategies and polynomial orders. It can be seen that the GERS minimizes the ndof compared to other refinements but does not yield satisfactory results in terms of convergence of the force. Moreover, this strategy demonstrates high sensitivity with regard to the order of the polynomial. The MS gives the best results, as the force value is less sensitive to the order of the space discretization and is in good agreement with the value obtained by means of the commercial FEM package Flux2D with a very dense second-order mapped mesh.

From the linear magnetoharmonic simulation, the eddy current distribution and losses are obtained. The reference value of the eddy current losses is obtained from the $h p$-convergence analysis under uniform refinement, which is presented in Fig. 5(a). The convergence of the eddy current losses, under adaptive refinement, is given in Fig. 6(a), while the corresponding computational time is shown in Fig. 6(b). The adaptive strategy stopping criteria are set to a maximum number of $5 \cdot 10^{3}$ dof and six mesh levels, which guarantees a reasonable solving time for the optimum mesh. This choice is necessary because the computational time of the hierarchical setting depends mainly on the level of the mesh, rather than the ndof. The computational time of the mesh selection for the magnetoharmonic problem, using MS adaptive refinements and biquartic THB-splines, is less than $90 \mathrm{~s}$ for the eddy 


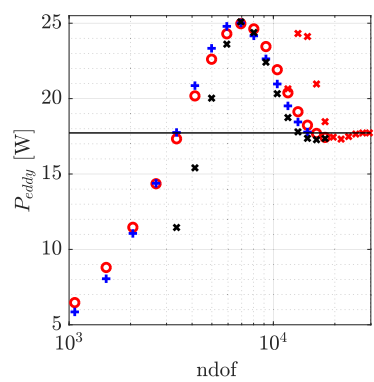

(a)

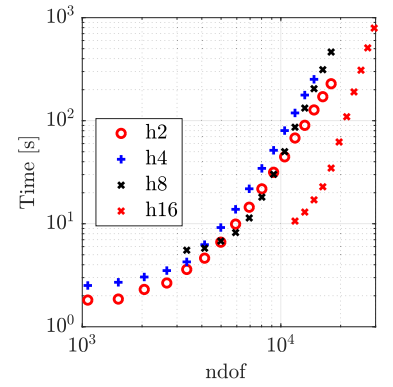

(b)
Fig. 5. Uniform $h p$-convergence analysis of (a) eddy current losses and (b) corresponding computational time. The black line represents the final value, which is also indicated in Fig. 6.

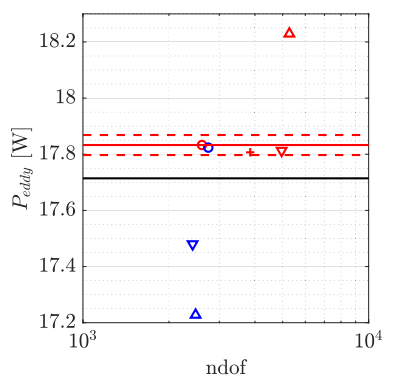

(a)

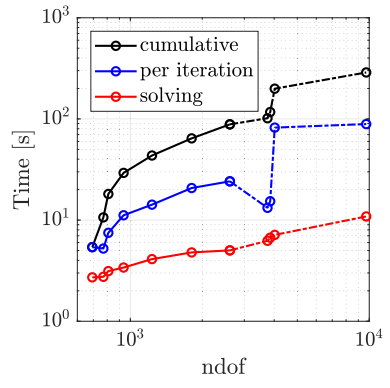

(b)
Fig. 6. (a) Eddy current losses convergence for the different strategies and orders, with the same legend as Fig. 4. The black line represents the reference value obtained from the $h p$-convergence analysis of Fig. 5, and the red dashed lines represent a variation of $0.2 \%$. (b) Computational effort using the adaptive MS refinements with biquartic THB-splines. The dotted-dashed lines give further indication of the trend although convergence has been reached.

current problem, while the solving time for the optimum mesh is about $5 \mathrm{~s}$. The tests were performed on the same machine and using a single core. Fig. 5(a) shows that under uniform $h$-discretizations, $p$-refinements ensure a smooth convergence toward a reference value. However, a large overshoot is observed, as the skin depth is orders of magnitude smaller than the thickness of the conductive part. Therefore, for solving with uniform refinements, the geometry of the conductive part would have to be truncated to a thickness of five times the skin depth to ensure smoother and faster convergence within a reasonable ndof, i.e., shifting the curve of Fig. 5(a) to the left as the skin depth over thickness ratio decreases. However, reducing the integration surface would inevitably lead to a small discrepancy on the eddy current loss estimation. Such a compromise is avoided by using the proposed adaptive method.

\section{DISCUSSION}

The function-based error estimate for the linear magnetoharmonic problems is reliable and demonstrates proper convergence of the global error estimate (gest). However, it is important to note that the convergence of the global error estimate does not imply the convergence of the global parameters (force or eddy current losses). For the nonlinear magnetostatic problem, such a local error estimate is not reliable for any refinement strategy, as the Newton-Raphson iterative solver would already minimize the residual to $10^{-18}$ at each iteration of the adaptive algorithm. In this paper, an error estimate based on the linear problem has instead been chosen to pursue the adaptive refinements, and the nonlinear solver has been used at the last iteration. Therefore, the selected optimum mesh, displayed in Fig. 1(b), is mostly refined in the air gap and around the corners of the geometry although the latter is less critical in the nonlinear problem because the saturation reduces the field divergence at the vertices. The adaptive strategy converges quickly to a precision of $0.1 \%$ and $0.2 \%$ for the force and eddy current losses values, respectively, which is sufficient for most engineering applications. Uniform and adaptive convergence analysis for a given problem and geometry are necessary to select the optimum discretization, in terms of both accuracy and computational effort. Moreover, it allows quantifying the solver precision, which, in turn, can be used to set the proper tolerance in an optimization problem.

\section{CONCLUSION}

Adaptive hierarchical refinement strategies enable the selection of an optimum mesh discretization for the considered problem. The hierarchical mesh discretization minimizes the ndof and, therefore, the memory requirements and computational effort while capturing the local field gradients and losses accurately. For complex geometries, the adaptive hierarchical refinement using the MS decreases the ndof by a factor 100 compared to FEM and by a factor 10 compared to uniform IGA while maintaining similar accuracy. Finally, the hierarchical adaptive method alleviates the need for geometrical model truncation and is advantageous in the case of multiscale problems.

\section{REFERENCES}

[1] K. Yamazaki, H. Mukaiyama, and L. Daniel, "Effects of multi-axial mechanical stress on loss characteristics of electrical steel sheets and interior permanent magnet machines," IEEE Trans. Magn., vol. 54, no. 3, Mar. 2018, Art. no. 1300304.

[2] E. M. Garau and R. Vázquez, "Algorithms for the implementation of adaptive isogeometric methods using hierarchical B-splines," Appl. Numer. Math., vol. 123, pp. 58-87, Jan. 2018.

[3] A. Buffa and E. M. Garau, "A posteriori error estimators for hierarchical B-spline discretizations," Math. Models Methods Appl. Sci., vol. 28, no. 8, pp. 1453-1480, 2018.

[4] A. Buffa and C. Giannelli, "Adaptive isogeometric methods with hierarchical splines: Error estimator and convergence," Math. Models Methods Appl. Sci., vol. 26, no. 1, pp. 1-25, 2016.

[5] R. Vázquez, "A new design for the implementation of isogeometric analysis in Octave and MATLAB: GeoPDEs 3.0," Comput. Math. Appl., vol. 72, no. 3, pp. 523-554, 2016.

[6] J. Gyselinck, L. Vandevelde, J. Melkebeek, and P. Dular, "Complementary two-dimensional finite element formulations with inclusion of a vectorized Jiles-Atherton model," COMPEL-Int. J. Comput. Math. Elect. Electron. Eng., vol. 23, no. 4, pp. 959-967, 2004.

[7] K. Bastiaens, D. C. J. Krop, S. Jumayev, and E. A. Lomonova, "Design and comparison of high-frequency resonant and non-resonant rotating transformers," in Proc. 21st Int. Conf. Elect. Mach. Syst. (ICEMS), Oct. 2018, pp. 1703-1708.

[8] S. Ausserhofer, O. Bíró, and K. Preis, "An efficient harmonic balance method for nonlinear eddy-current problems," IEEE Trans. Magn., vol. 43, no. 4, pp. 1229-1232, Apr. 2007.

[9] A. Buffa and E. M. Garau, "Refinable spaces and local approximation estimates for hierarchical splines," IMA J. Numer. Anal., vol. 37, no. 3, pp. 1125-1149, 2017.

[10] W. Dörfler, "A convergent adaptive algorithm for Poisson's equation," SIAM J. Numer. Anal., vol. 33, no. 3, pp. 1106-1124, 1996.

[11] C. Bracco, C. Giannelli, and R. Vázquez, "Refinement algorithms for adaptive isogeometric methods with hierarchical splines," Axioms, vol. 7 , no. 3 , p. $43,2018$. 